\title{
Radio frequency identification based smart parking system using Internet of Things
}

\author{
Rahman Atiqur \\ School of Information and Communication Engineering, Chongqing University of Posts and Telecommunications, \\ Chongqing Key Lab of Mobile Communications Technology, P.R. China \\ University of Chittagong, Faculty of Engineering, Computer Science and Engineering Department, Bangladesh
}

\begin{tabular}{l} 
Article Info \\
\hline Article history: \\
Received Jul 14, 2020 \\
Revised Aug 4, 2020 \\
Accepted Oct 20, 2020 \\
\hline
\end{tabular}

\section{Keywords:}

GSM module Internet of Things

Raspberry Pi 4

RFID

Ultrasonic sensor

\begin{abstract}
The ideas of shrewd urban communities have consistently been a fantasy. There have been headways produced using the recent years to make a shrewd city dream to reality with the advancement of innovation, smart gadgets are getting increasingly normal in regular daily existence. In this paper a radio frequency identification (RFID) based smart parking system using Internet of Things (IoT) innovation is actualized. The ultrasonic sensors are set before the parking spots and speaker is utilized for sign. On the off chance that the parking space is vacant, at that point light emitting diode (LED) will squint and the parking opening is full LED will be OFF. At the point when vehicle is left the RFID will peruse the data and cut the particular sum and by utilizing IoT location will be sent through short message service (SMS) to proprietor using global system for mobile communications (GSM) and global positioning system (GPS) advancements.
\end{abstract}

This is an open access article under the $\underline{C C B Y-S A}$ license.

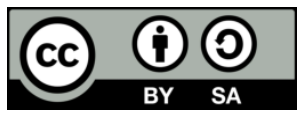

\section{Corresponding Author:}

Rahman Atiqur

School of Information and Communication Engineering

Chongqing University of Posts and Telecommunications

Key Lab of Mobile Communications Technology, Chongqing, 400065, P. R. China

Email: bulbul.cse.cu@gmail.com

\section{INTRODUCTION}

The Internet of Things (IoT) can move information through the system without including human connections. IoT permits clients to utilize reasonable remote innovation and furthermore encourages the client to move the information into the cloud. IoT encourages the client to look after straightforwardness. The possibility of IoT began with the personality of things for associating different gadgets. These gadgets can be controlled or observed through PCs over the web. IoT contains two unmistakable words "Inter" and "Things", where the Internet is a huge system for interfacing servers with gadgets [1]. Inter empowers the data to be sent, get or even speak with the gadgets. The parking issue causes air contamination and traffic blockage [2]. In the present situation, parking spots are difficult to look in an everyday life for the individuals. As per the ongoing review, there will be a fast increment in the vehicle's populace of over 1.6 billion around 2035 [3]. Around one million barrels of world's oil is being scorched ordinary [4]. Hence, a smart parking framework is the key answer for lessening the waste phase of the fuel. The answer for the issues is being raised. Smart parking can be an answer for limiting a client's time and effectiveness just as the general expense of the fuel consumed looking for the parking spot. In this, the information is gathered from the sensor and through dissecting and preparing, the yield is obtained [5]. This information gets transmitted in the gadgets which extricates the applicable data and sends it to the raspberry pi 4 gadgets which provide the order guidance for the information to the specific gadgets all the while. Raspberry pi 4 imparts the sign to the servo engine 
alongside GSM module which further gives guidelines and notice to the client. At the point when the client enters the parking zone, RFID card dispensed to the enrolled client is filtered by the reader module subsequently guaranteeing the security of the client personality. This empowers the client to get the data of the accessible parking spot just as SMS notice to the enrolled client's portable number. It comprises three sections where the initial segment is the parking area which incorporates raspberry pi 4 gadgets alongside ultrasonic sensor. The client connects with the parking area with the assistance of these gadgets. The second segment of the paper incorporates the cloud web administrations which go about as a mediator between the client and the vehicle parking area. The cloud is refreshed by the accessibility of the parking area. The cloud administration is regulated by the administrator yet it can likewise be seen by the client to check the accessibility. The third area of the paper is the client side. The client gets told for the accessibility by means of SMS through the GSM module. The client collaborates with the cloud just as a parking area. The client gets the notice when the parking accessibility is full which spares the ideal opportunity for the client. In section 2 We study some literature. Section 3 presents proposed smart car parking system model and describes how they work. Section 4 gives the result based on proposed model and describes how these systems are fruitful in our daily life and section 5 concludes the paper.

\section{LITERATURE REVIEW}

The sensors utilized in IoT based smart parking system stores and gets information from remote areas with the assistance of the cloud these variables offer raise to cloud of things (COT). The hubs could be observed and controlled from any area the framework that we propose gives data in regards to the accessibility of the parking slots with the assistance of the versatile application the clients from the remote area can book the parking slots [6]. A calculation is utilized to build effectiveness of cloud-based parking framework and system engineering innovation is utilized. This calculation is utilized to locate the least cost parking spot. Considering the quantity of parking spots accessible and furthermore considering the separation of the parking spot from the client. The client can legitimately get to the cloud-based server and discover the data on the parking spot. The client can likewise introduce an application in their cell phones to get to this data. With the assistance of this calculation, holding up time of the client to discover a parking spot can be limited. Security perspectives are excluded from this paper [7]. A remote sensor hub alongside advanced mobile phone application is being utilized to discover the parking spot. Since, remote innovation is utilized here the framework has high precision and effectiveness.

In this framework, installed units are utilized to speak with different vehicles. The client leaves his vehicle in any of the few bayous accessible mechanical lift lifts the vehicle out. A ticket key and id are given to the client and it is just known to the client which is utilized to recover the vehicle. The client need not convey any paper ticket since a RFID card is given to the client. The innovation utilized here is efficient. Security highlights must be improved to ensure the client' protection [8]. The creator of smart parking framework the study has separated identifier framework and vehicle sensors into two math classes as meddling sensors and non-nosy sensors. Meddling sensors are introduced in openings out and about the surface by burrowing under the street. Non-meddling sensors don't influence the outside of the street and it very well may be effortlessly introduced and kept up. Smart parking framework causes us to determine the establishing issues of the traffic blockage and it likewise decreases the outflow from a vehicle. [9] A paper proposes an effective approach to unfurl the issue of parking accessibility in the ongoing situation and to decrease the time utilization. In this, the information is sent locally with gadgets which channel the information. This sign is transmitted over the cloud for the procedure just as for assessment which uses AI calculations. This paper utilizes a cell phone application that associates the client with the constant traffic status through Google API. In this way, traffic blockage is kept away from. This paper doesn't give the booking office to the car parking [10].

\section{PROPOSED SYSTEM}

The following Figure 1 shows the block diagram of the proposed system. The entire system is controlled by the Raspberry Pi 4 device. In this proposed system mainly RFID Reader, ultrasonic sensors, GSM module, GPS unit, LED display, and the speaker is used. From Figure 1, we see that Raspberry pi 4 is attached with a power supply for running. When the system needs to run, trigger the power supply switch on. When the system faces difficulty reset the system using the reset button which is a great feature in the proposed system. Each component of the proposed system is explained in a detailed manner in the subsequent sections. From the subsequent section, anyone can get an idea about the components used and also know how they work.

IAES Int J Rob \& Autom, Vol. 10, No. 1, March 2021 : $41-50$ 


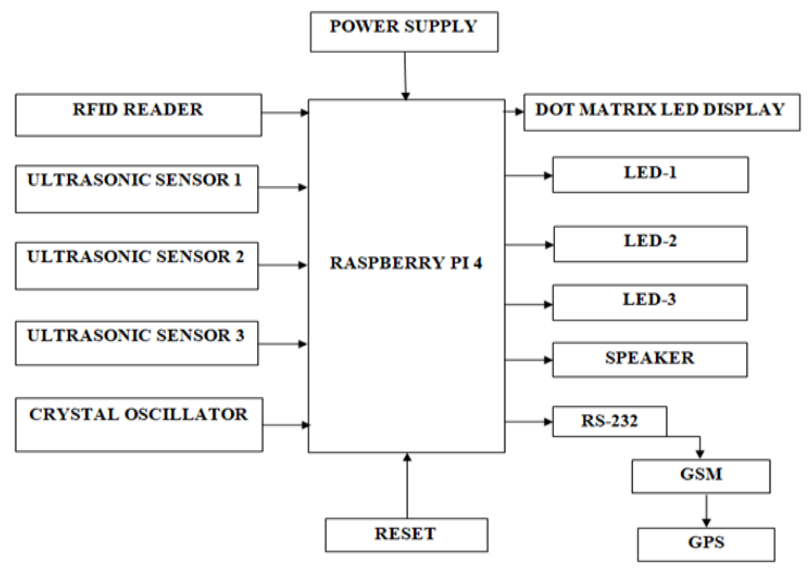

Figure 1. Block diagram of proposed smart parking system using Raspberry Pi 4

\subsection{Raspberry Pi 4}

Raspberry Pi 4 is a debit card sized single-board computer with an OS Raspbian installed [11, 12]. Figure 2 shows the available interfaces in Raspberry Pi 4 [12].The Raspberry collects the number of parking slots from each NodeMCU V3 and processes the data and updates the database server with a total number of vacant slots. Raspberry Pi 4's specifications are specified in Table 1.

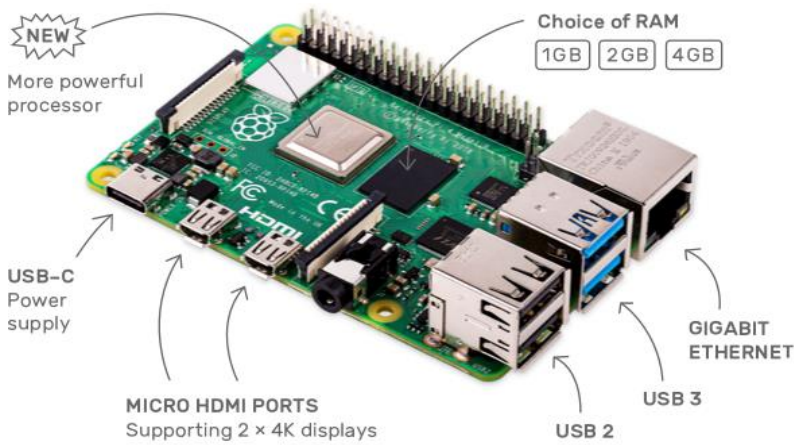

Figure 2. Raspberry Pi4 boards

Table 1. Raspberry Pi 4 specification [13]

\begin{tabular}{ll}
\hline Specifications & Values \\
\hline CPU & Broadcom BCM2711, Quad core Cortex-A72 (ARM v8) 64-bit SoC @ 1.5GHz \\
RAM & 1GB, 2GB or 4GB LPDDR4-2400 SDRAM (depending on model) \\
USB Ports & 2 USB 3.0 ports; 2 USB 2.0 ports \\
GPIO Pins & Raspberry Pi standard 40 pin \\
Other Features & 1. HDMI - 2 × micro-HDMI ports (up to 4kp60 supported) \\
& 2. Display port - 2-lane MIPI DSI \\
& 3. Camera port - 2-lane MIPI CSI \\
& 4. Audio - 4-pole stereo audio and composite video port \\
& 5. Storage - Micro-SD card slot for loading operating system and data storage \\
& 6. Misc - H.265 (4kp60 decode), H264 (1080p60 decode, 1080p30 encode), OpenGL ES 3.0 graphics \\
& 7. OS-Debian Linux 10 based
\end{tabular}

\subsection{Ultrasonic sensor}

The theory behind the ultrasonic parking sensor is based on echo-location. The frequency of the sound is so high that men cannot perceive it, which is useful because it provides correctness and remains ordinary. As sound hits a solid object, after creating an echo it is reflected back. Since the speed of sound is well-known and steady for similar conditions, it is possible to decide the distance of the object you hear an echo from by multiplying the speed of sound by half the time it takes to hear the echo (because the echo time 
is actually the time it takes the sound to go there and back) [14]. From Table 2 we see that the detecting range of an ultrasonic parking sensor lies between 0.3 to 2 meter, the beam width of an ultrasonic parking sensor above 60 degree in both horizontally and vertically, the beam pattern of an sensor is canonical, and the frequency ranges from 315 megahertz to 433 megahertz as well as unit cost of an ultrasonic parking sensor is near about 30 INR. Figure 3 shows an ultrasonic parking sensor that is used in my system for detecting the vacant slot. It detects a parking slot based on the aforementioned echo-location mechanism. This mechanism is very strong and makes my system more promising.

Table 2. Technical specification of sensors [15]

\begin{tabular}{lc}
\hline \multicolumn{1}{c}{ Parameters } & Ultrasonic parking sensor \\
\hline Range & $0.3 \sim 2 \mathrm{~m}$ \\
Beam-width & $\mathrm{H}>60, \mathrm{~V}>60$ degree \\
Beam Pattern & Conical \\
Frequency & $315 \mathrm{MHz} / 433 \mathrm{MHz}$ \\
Unit Cost & $30 \mathrm{INR}$. \\
\hline
\end{tabular}

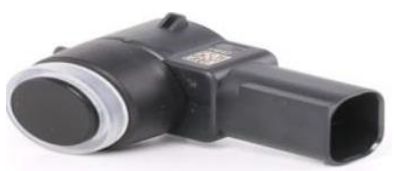

Figure 3. An ultrasonic parking sensor

\subsection{Radio frequency identification reader}

Radio-frequency identification (RFID) utilizes electromagnetic fields to consequently recognize and follow labels appended to objects [16]. A RFID label comprises a minuscule radio transponder; a radio beneficiary and transmitter. When activated by an electromagnetic cross examination beat from a close by RFID per user gadget, the tag transmits advanced information, normally a distinguishing stock number, back to the reader. This number can be utilized to stock merchandise. There are two sorts. Latent labels are controlled by vitality from the RFID reader's cross examining radio waves. Dynamic labels are fueled by a battery and in this manner can be read at a more prominent range from the RFID reader; up to many meters. Not at all like a standardized tag, shouldn't the tag be inside the view of the reader, so it might be inserted in the following object. RFID is one strategy for programmed automatic identification and data capture (AIDC). RFID labels are utilized in numerous businesses. For instance, a RFID label appended to a vehicle during creation can be utilized to keep tabs on its development through the mechanical production system; RFIDlabeled pharmaceuticals can be followed through distribution centers; and embedding RFID microchips in domesticated animals and pets empowers positive recognizable proof of creatures [16]. Figure 4 displays a RFID card reader. RFID card reader functions in the above mentioned descriptions. This RFID card reader is used in my recommended system for detecting the number plate of a car. Reading the number plate of a car the admin can confirm the arrived car is the previously booked one or not. If the number plate does not match then the car is not allowed for parking.

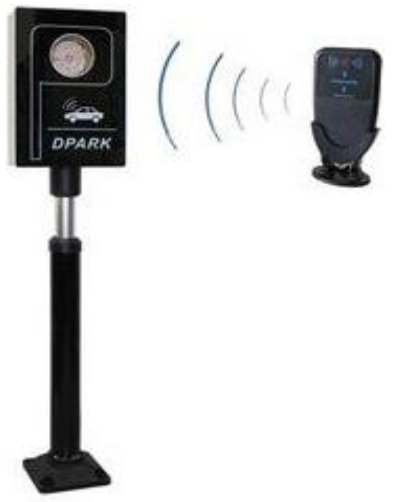

Figure 4. RFID card reader 


\subsection{Global system for mobile communications}

Global system for mobile communication known as (GSM) is a design utilized for versatile correspondence in a large portion of the nations. This module is utilized to set up correspondence between a PC and a GSM-GPRS framework, it comprises a GSM/GPRS modem gathered along with power flexibly circuit and correspondence interfaces (like RS-232, USB, and so forth.) for PC. Worldwide Packet Radio Service (GPRS) is an expansion of GSM that empowers higher information transmission rate. The activity can be performed by GSM module is as per the following:

- Receive, reject and make a voice call.

- Receive, send and erase messages in a SIM.

- Add, look and get phonebook passages of the SIM.

Global system for mobile communications (GSM) modems are specific kinds of modems that work over membership based remote systems, like a cell phone. A GSM modem acknowledges a subscriber identity module (SIM) card, and essentially acts like a cell phone for a PC. Such a modem can even be a committed cell phone that the PC utilizes for GSM organize abilities [17]. Figure 5 shows a typical GSM module. From Figure 5 we see that a typical GSM module is built with an antenna, audio connector, buzzer, SIM card holder, supply port as well as a communication port. Using these GSM modules, a short message was sent to the proprietor.

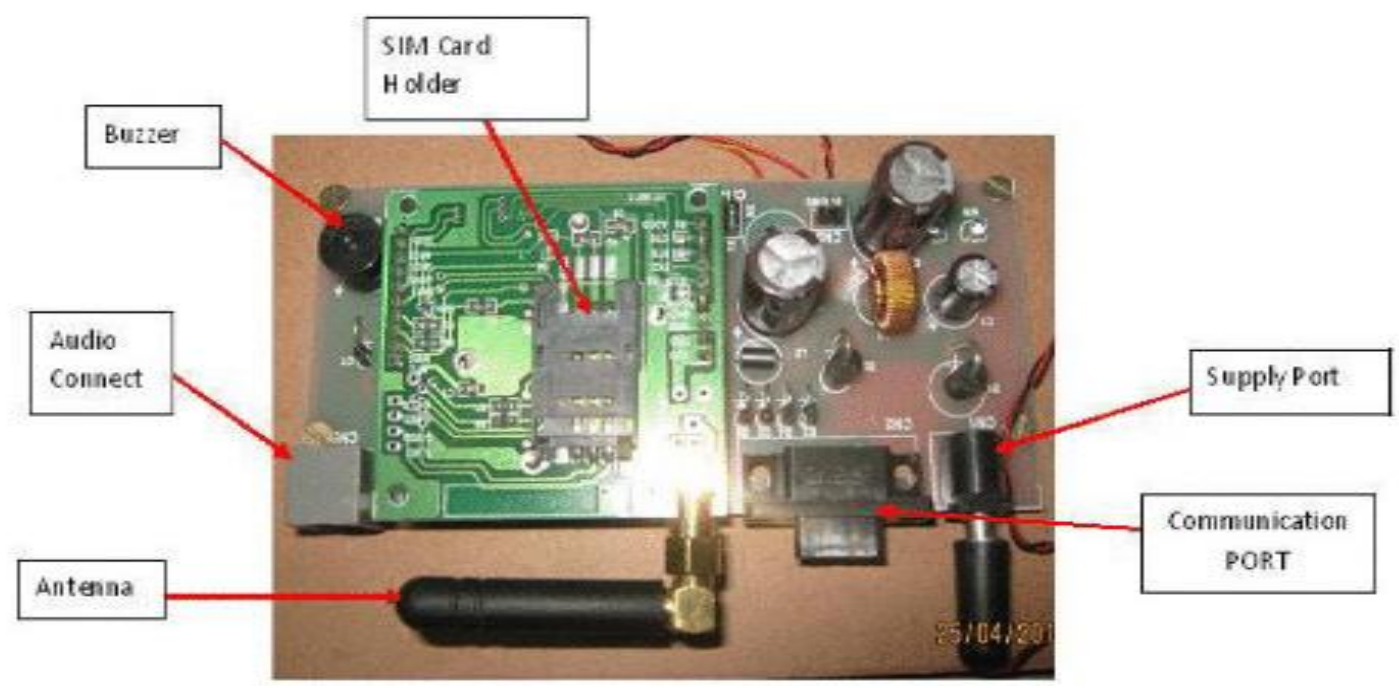

Figure 5. Typical GSM module

\subsection{Global positioning system}

The global positioning system (GPS) is a U.S. space-based worldwide route satellite framework. It gives solid situating, route, and timing administrations to overall clients on a persistent premise in all climate, day and night, anyplace on or close to the Earth. PS gets the situation of the vehicle and remote correspondence sends it to the server. A cell phone utilizes for clients to remotely see the stopping, status of spots and book an opening physically. The proposed framework diminishes the looking through time of free space by offering courses to free opening.

\subsection{Speaker}

A speaker or beeper is a sound flagging gadget, which might be mechanical, electromechanical, or piezoelectric. Common employment of speakers and beepers incorporate alert gadgets, clocks. Early gadgets depended on an electromechanical framework indistinguishable from an electric ringer without the metal gong. A speaker associated with raspberry pi 4 gadget is actuated when the parking garage is full. It shows the accessible space in the parking garage on LED. Thus the time taken to check the accessible space is diminished. The framework can be introduced at passageway and exit.

\subsection{Light emitting diode display}

A LED show is a level board show that utilizes a variety of light-producing diodes as pixels for a video show. Their brilliance permits them to be utilized outside where they are noticeable in the sun for store signs and bulletins. As of late, they have additionally gotten ordinarily utilized in goal signs on open

Radio frequency identification based smart parking system using Internet of Things (Rahman Atiqur) 
transport vehicles, just as factor message signs on parkways. Driven presentations are fit for giving general enlightenment notwithstanding visual showcases, as when utilized for stage lighting or other beautifying (instead of instructive) purposes. LED showcases can offer higher complexity proportions than a projector and are in this way an option in contrast to conventional projection screens, and they can be utilized for huge, continuous (without a noticeable lattice emerging from the bezels of individual presentations) video dividers. miniaturized scale LED shows are LED shows with littler LEDs, which presents huge improvement challenges. LED Display (light-emitting diode show) is a screen show innovation that utilizes a board of LEDs as the light source. Right now, an enormous number of electronic gadgets, both little and huge, use LED show as a screen and as an association medium between the client and the framework. Present day electronic gadgets, for example, cell phones, TVs, tablets, PC screens, workstations screens, and so on, utilize a LED show to show their yield [18].

LED Display is one of the principle screen shows that are as a rule economically utilized. The greatest favorable position of the LED show is its productive and low-vitality utilization, which is particularly required for handhelds and chargeable gadgets, for example, cell phones and tablets. A LED show comprises various LED boards that, thus, comprise of a few LEDs. LEDs have various favorable circumstances over other light-transmitting sources that can be utilized then again. Besides being power effective, LEDs produce more splendor and more noteworthy light force. LED-Display is not the same as the vacuum fluorescent showcase utilized in some purchaser hardware, for example, vehicle sound systems, videocassette recorders, and so forth, and, consequently, these two ought not to be mistaken for one another.

Figure 6 shows the 7 segment display's pin diagram. Here I placed two pin configuration's view of a typical 7 segment display. From Figure 6 we clearly see that this display shows the status of a parking lot in real-time. Users can easily reach a decision by observing the parking status on the display board. Table 3 summarizes the 7 segment display pin configuration. From Table 3 we see that the 7 segment displays have E through $\mathrm{G}$ total of ten pins and different pins have different functions. For example, pin E controls the left bottom LED of the 7-segment display. Another pin has another function that we have seen in Table 3.
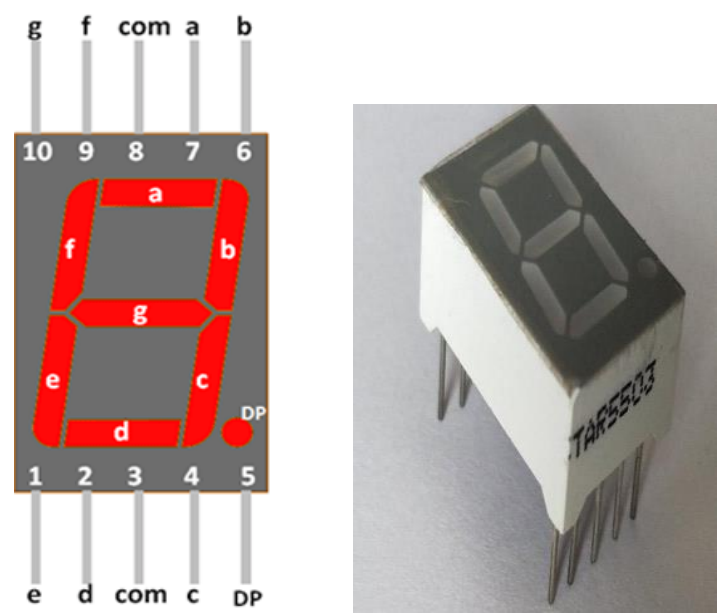

Figure 6. 7 Segment display pin diagram

Table 3. 7 Segment display pin configuration

\begin{tabular}{ccl}
$\begin{array}{c}\text { Pin } \\
\text { Number }\end{array}$ & $\begin{array}{c}\text { Pin } \\
\text { Name }\end{array}$ & Description \\
\hline 1 & E & Controls the left bottom LED of the 7-segment display \\
2 & D & Controls the bottom most LED of the 7-segment display \\
3 & Com & Connected to Ground/Vcc based on type of display \\
4 & C & Controls the right bottom LED of the 7-segment display \\
5 & DP & Controls the decimal point LED of the 7-segment display \\
6 & B & Controls the top right LED of the 7-segment display \\
7 & A & Controls the top most LED of the 7-segment display \\
8 & Com & Connected to Ground/Vcc based on type of display \\
9 & F & Controls the top left LED of the 7-segment display \\
10 & G & Controls the middle LED of the 7-segment display \\
\hline
\end{tabular}

IAES Int J Rob \& Autom, Vol. 10, No. 1, March 2021: $41-50$ 


\section{RESULTS}

From Figure 7 we can see that my recommended design consists of hardware components like Raspberry pi 4, ultrasonic sensors, LED display, RFID module, Speaker, GSM module as well as parking servo motor. All devices are connected all together form my recommended system that are cost effective, fuel savings, faster constructions, profitable, reduced maintenance cost, tax independent as well as risk free too.

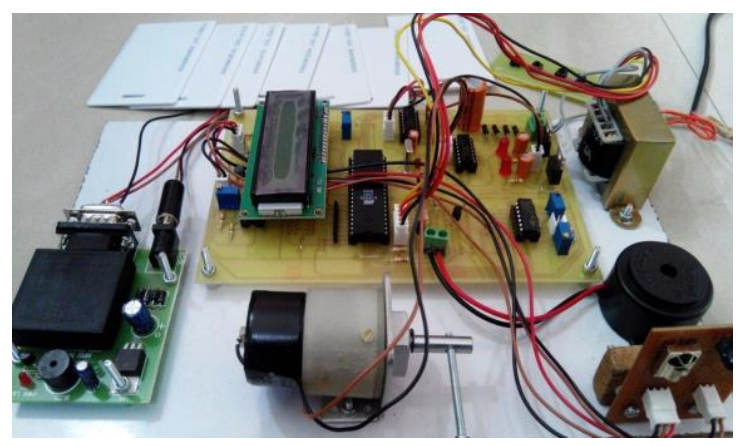

Figure 7. RFID based smart car parking system

\subsection{Economical benefits of automated car parking system}

\subsubsection{More profitable land use}

Automated parking systems require essentially less territory and volume for a given number of parking spots than other parking alternatives. Automated Parking frameworks empowers the more productive utilization of important land for inhabitants, green space and so forth and give property designers different alternatives, for example, limiting the region required for parking to expanding the quantity of parking spots or some ideal point in the middle of the two.

\subsubsection{Parking optimized for profitability}

Regular parking arrangements are excessively enormous or unfeasible though the structure adaptability of automated parking systems permits them to fit in areas or zones. Automated parking frameworks can be introduced inside, under or between existing structures, thin and profound regions and even sporadically formed spaces: on a level plane, vertically or both. Automated Parking frameworks help increment benefit by utilizing unusable or lower esteem space for vehicle parking [19]. Figure 8 displays how my recommended smart parking system minimizes searching time. From the above figure we can see that the vertical line displays the distance travelled measurements and the horizontal line shows the required searching time in minutes. From the figure we see that my recommended system takes less searching time when compared to other existing systems. The yellow line is my recommended system's searching time measurement and it is clear that my system is the best one.

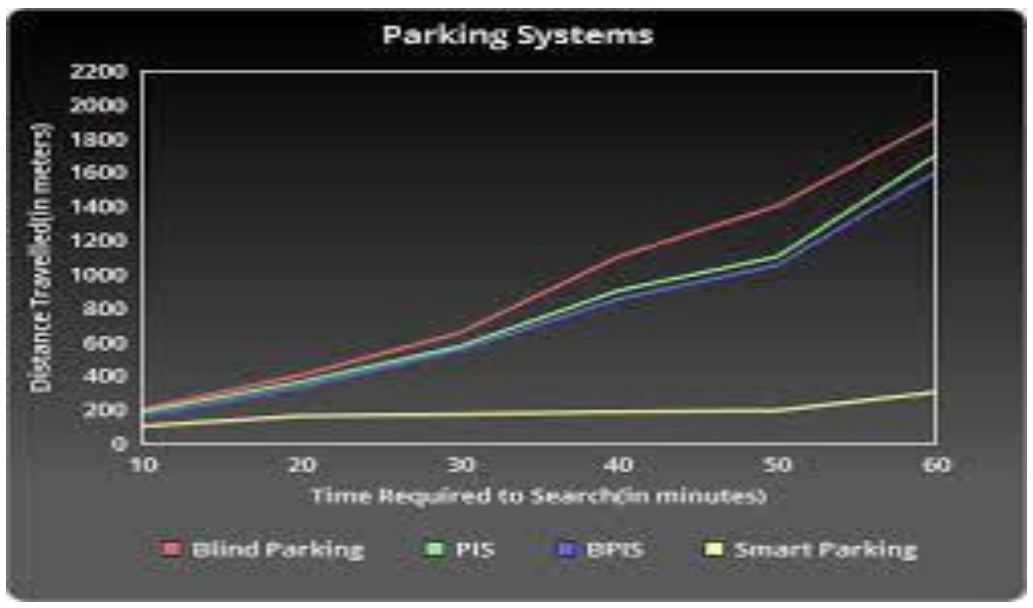

Figure 8. Smart parking minimizing searching time 


\subsubsection{Capital cost}

The regular thought that the automated parking frameworks consistently cost more than multi-story parking structures is excessively oversimplified and as often as possible erroneous. Automated parking frameworks can be substitutions for regular vehicle parking. Automated parking frameworks' considerably smaller size and structure adaptability can essentially move capital expense and task productivity for if the engineers join them into starter plans.

\subsubsection{Reduced fuel and maintaining costs}

Activity and upkeep costs are exceptionally explicit to every application. Mechanized leaving frameworks have the upside of requiring no or insignificant lighting, ventilation, fire concealment, observing, tidy up, staff and safety efforts in the empty leaving territory not at all like the regular vehicle park. The following figure graphically shows how fuel consumptions are ensured [20]. Figure 9 shows the fuel consumption visualization of my recommended system. From the figure we see that distance is measured in meters and it is placed in the horizontal axis as well as the fuel rate is placed in the vertical axis. The red line in the figure is my proposed system's fuel consumption rate and it is smaller when compared with the VTCPFM fuel consumption rate.

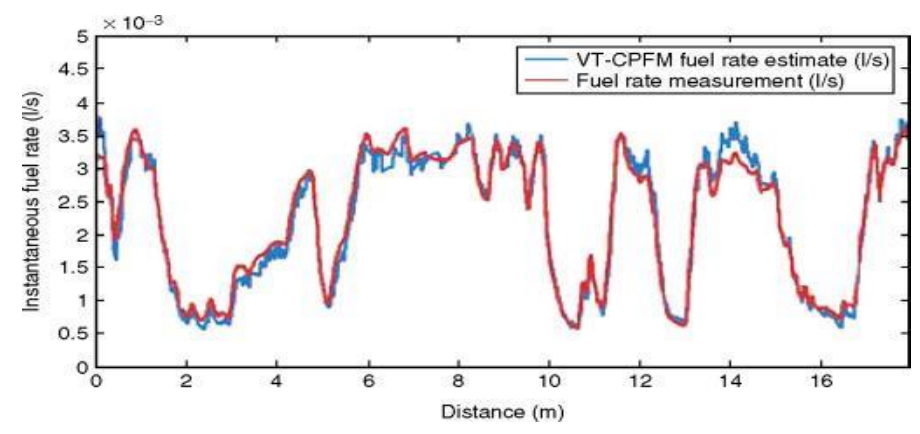

Figure 9. Shows the fuel consumptions

\subsubsection{Sustainable building}

Automated parking systems offer various favorable circumstances over regular parking with regards to green space, emanations and carbon impression decrease, sunlight based access, storm water spillover and that's just the beginning. Greenhouse gases (VOCs, CO, NO, and so forth.) can be diminished 60 to 85 percent since no driving is required to discover spaces and park cars. Permit increasingly green space by lessening land necessities. Automated parking frameworks can be considerably more vitality productive than ordinary parking arrangements, particularly in underground or encased parking applications. Less exhuming profundity of automated parking frameworks limits the potential groundwater sway. Unlike the regular vehicle parking Automated parking frameworks require substantially less structure material. Automated parking frameworks can limit the impediment of perspectives and daylight. Recycling of high worth structure materials, for example, steel.

\subsubsection{Inherent securities}

The automated parking systems idea inalienably gives a lot more significant levels of assurance and security for vehicles, their substance and their drivers. Vandalism and robbery are for all intents and purposes unimaginable in an Automated parking frameworks. Individual security is a lot higher than in vehicle leaves since drivers and travelers are consistently in well-lit, profoundly noticeable/open sections and leave regions at road level. Robotized stopping frameworks are likewise a perfect answer for the impaired since passage and leave covers can promptly oblige explicit prerequisites and construction standards.

\subsubsection{Lower risk and liability}

Protection premiums are regularly intensely impacted by the likelihood of mishaps or different occasions happening; here automated parking frameworks may help as automated parking frameworks limit the potential for property harm, burglary, individual injury or demise. The chance of marks, scratches, other harm and vandalism to vehicles, burglary of property from vehicles, vehicle burglary, theft, pyromania, fire, ambush, assault, falls and self-destruction can be decreased significantly by utilizing the intrinsic well-being and security of automated parking frameworks. 


\subsubsection{Tax advantages}

The property designers may have huge assessment favorable circumstances as quickened devaluation contrasted with vehicle parks or parking structures by utilizing Automated parking frameworks. A few nations grant quicker, or quickened, devaluation rates for gear dependent on its helpful life as hardware esteem decays at a quicker rate in the previous years. Automated parking systems might be qualified for a lot higher devaluation rates, expanding benefits contrasted with the 25 to multiyear deterioration of structures, for example, and car parks.

\subsubsection{Faster constructions}

Automated parking systems are normally quicker and simpler to introduce in view of a lot fewer and profoundly pre-manufactured structures, than bigger solid vehicle leaves. There is significantly less volume to exhume and move, this prompts decreased development costs, less enthusiasm during development and a quicker beginning of income age.

\subsubsection{Improved profitability and other factors}

Less quantifiable advantages of automated parking system that can improve productivity for engineers include [20]:

- Location: Automated parking frameworks can add to the estimation of the area as it can pick up individuals' consideration.

- $\quad$ Reduced visual effect: By utilizing automated parking systems we can lessen the space parking slot that can decrease rent/rental rates the lower statutes and littler volumes of automated parking system limit check of perspectives and daylight.

- $\quad$ Aesthetics: Automated parking system to mix in to the environmental factors as it has adjustable outside medicines. Automated parking systems give property designers various advantages and opportunities for vehicle leaving, so an adjustment in believing is expected to exploit automated parking systems.

\section{CONCLUSION}

The headway of the IoT and cloud innovations has offered ascend to the additional opportunities as far as brilliant urban areas. Shrewd parking offices have consistently been the center of developing smart urban communities. The framework gives a constant procedure and data of the parking spaces. This paper improves the presentation of sparing clients time to find a proper parking spot. It assists with settling the developing issue of traffic clogs. With respect to the future work the clients can book a parking spot from a remote area.

\section{ACKNOWLEDGEMENTS}

I would like to convey my heartfelt gratitude towards my guide, Professor Dr. Yun Li for his constant guidance, encouraging help and inspiring words. I am thankful to the School of Information and Communications Engineering for their support.

\section{REFERENCES}

[1] A. O. Kotb, Y. Shen and Y. Huang, "Smart Parking Guidance, Monitoring and Reservations: A Review," in IEEE Intelligent Transportation Systems Magazine, vol. 9, no. 2, pp. 6-16, Summer 2017, doi: 10.1109/MITS.2017.2666586.

[2] S. Shinde, A. Patil, S. Chavan, S. Deshmukh and S. Ingleshwar, "IoT based parking system using Google," 2017 International Conference on I-SMAC (IoT in Social, Mobile, Analytics and Cloud) (I-SMAC), Palladam, 2017.

[3] H. Chaudhary, P. Bansal and B. Valarmathi, "Advanced CAR parking system using Arduino," 2017 4th International Conference on Advanced Computing and Communication Systems (ICACCS), 2017, pp. 1-5.

[4] N. R. N. Zadeh and J. C. Dela Cruz, "Smart urban parking detection system," 2016 6th IEEE International Conference on Control System, Computing and Engineering (ICCSCE), Batu Ferringhi, 2016, pp. 370-373, doi: 10.1109/ICCSCE.2016.7893601.

[5] P. Sheelarani, S. P. Anand, S. Shamili and K. Sruthi, "Effective car parking reservation system based on internet of things technologies," 2016 World Conference on Futuristic Trends in Research and Innovation for Social Welfare (Startup Conclave), Coimbatore, pp. 1-4, 2016.

[6] Prof. Yashomati R. Dhumal, Harshala A. Waghmare, Aishwarya S. Tole, Swati R. Shilimkar, "Android Based Smart Car Parking System," International Journal of Advanced Research in Electrical, Electronics and Instrumentation Engineering IJAREEIE, vol. 5, no. 3, pp-1371-1374, 2016.

[7] Faiz Ibrahim Shaikh, Pratik NirnayJadhav, Saideep Pradeep Bandarakar, "Smart parking system based on embedded system and sensor network," Smart Parking System Based on Embedded System and Sensor Network. International Journal of Computer Applications IJCA, vol. 140, pp.45-51 2016. 
[8] R. Garra, S. Martínez and F. Sebé, “A Privacy-preserving pay-by-phone parking system,” IEEE Transactions on Vehicular Technology, vol. 66, no. 7, pp. 5697-5706, Jul 2017.

[9] F. Wang, L. Yang, J. Yang, Y. Zhang, S. Han and K. Zhao, "Urban intelligent parking system based on the parallel theory," 2016 International Conference on Computing, Networking and Communications, 2016.

[10] Amala, Ch, G. Naga Lakshmi, Ksri Devi, G. Meghana, and G. Thabitha. "Iot based smart parking management system using RFID technology," International Journal for Recent Developments in Science \& Technology, vol. 4, no. 6, pp. 142-147, June 2020.

[11] Atiqur, Rahman, and Ali Md Liton, "Automated smart car parking system using internet of things (IoT) technology for smart cities demand," North American Academic Research, vol. 3, no. 8, pp. 7-19.

[12] Raspberry Pi 4 Documentation, [Online]. Available: https://www.raspberrypi.org/.

[13] Raspberry Pi4 Configuration, [Online]. Available: http://www.databook.bz/?cat=56".

[14] A. Dimitrov and D. Minchev, "Ultrasonic sensor explorer," 2016 19th International Symposium on Electrical Apparatus and Technologies (SIELA), 2016.

[15] Parking Sensors, [Online]. Available: https://www.parkingsensors.co.uk/page/technical-specifications.

[16] Rahman Atiqur, Yun Li, "Automated smart car parking system using raspberry Pi 4 and iOS application;" International Journal of Reconfigurable and Embedded Systems IJRES, vol. 9, no. 3, pp. 229-234, 2020.

[17] Rahman Atiqur, Guangfu Wu, Ali Md Liton, "Mobile edge computing for internet of things (IoT): security and privacy issues," Indonesian Journal of Electrical Engineering and Computer Science IJEECS, vol. 18 no.3, pp.1486-1493, 2020, doi: http: 10.11591/ijeecs.v18.i3.pp1486-1493.

[18] Rahman Atiqur, Ali Md Liton, "Content Caching Strategy at Small Base Station in 5G Networks with Mobile Edge Computing," International Journal of Science and Business, vol. 4, no. 4, pp. 104-112, 2020, doi: 10.5281/zenodo.3746233.

[19] Yacine Atif, Jianguo Ding, Manfred A. Jeusfeld "Internet of things approach to cloud-based smart car parking," Procedia Computer Science, vol. 98, pp. 193-198, 2016.

[20] Chandi Patel, Monalisa Swarni, Priya Saxena, Sejal Shah, "Rotary automated car parking system," International Journal of Engineering Science and Innovative Technology IJESIT, vol. 4, no. 2, pp. 408-415, 2015.

\section{BIOGRAPHY OF AUTHOR}

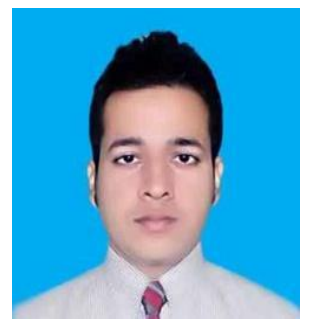

Mr. Rahman Atiqur received his Bachelor of Science (B. Sc) and Master of Engineering (M. Eng.) degree from the Department of Computer Science and Engineering at the University of Chittagong, Chittagong, Bangladesh. In profession, he worked in the Department of Computer Science and Engineering, University of Chittagong, Bangladesh as an Assistant Professor from April 2016. Formerly he was a lecturer in the Department of Computer Science and Engineering, University of Chittagong, Bangladesh. He is now conducting his Ph.D. research works under the Chinese Government Scholarships (CGS) Program at Chongqing University of Posts and Telecommunications, Chongqing, China. His current research interest lies in the field of edge computing-based IoT systems. 